\title{
A Subspace-based Method for DOA Estimation of Uniform Linear Array in the Presence of Mutual Coupling
}

\author{
B. Liao, Z. G. Zhang and S. C. Chan \\ Department of Electrical and Electronic Engineering \\ The University of Hong Kong, Pokfulam Road, Hong Kong \\ \{liaobin, zgzhang, scchan\}@eee.hku.hk
}

\begin{abstract}
This paper develops a subspace-based method for direction-of-arrival (DOA) estimation of uniform linear array (ULA) in the presence of mutual coupling. As the mutual coupling coefficient between two sensor elements is inversely related to their separation and is negligible when they are separated by a few wavelengths, the mutual coupling matrix (MCM) of a ULA can be well approximated as a banded symmetric Toeplitz matrix, which greatly reduces the number of unknown parameters to be estimated. Using the subspace principle, we propose a new method for joint estimation of the DOAs of incoming signals and banded symmetric Toeplitz MCM by reconstructing the steering vector to a specific matrix form. The proposed method achieves a better performance especially for weak signals than the method in [8], since the whole array, instead of the middle subarray in [8], is used for DOA estimation. Simulation results illustrate that both DOAs and mutual coupling coefficients can be estimated efficiently with the proposed method.
\end{abstract}

\section{INTRODUCTION}

Mutual coupling, which is caused by the interaction among the array elements, may seriously degrade the performance of high-resolution direction finding techniques such as MUSIC [1] and ESPRIT [2]. In ideal situations, the steering vector is assumed to be exactly known which depends on the array geometry and the signal location. However, such an assumption is far from reality, as the steering vector in real systems may be distorted by impairments such as mutual coupling, array gain/phase uncertainties and sensor position perturbation. Since the presence of mutual coupling would lead to considerable deterioration in direction finding of conventional high-resolution direction-of-arrival (DOA) estimation algorithms, mutual coupling calibration has received extensive attention [3-8].

One kind of methods for mutual coupling calibration makes use of extra sources with known locations, namely, calibration sources. The maximum-likelihood (ML)-based method proposed in [3] can compensate for mutual coupling, array gain/phase uncertainties as well as sensor position errors by applying calibration sources. Unfortunately, it may be difficult to obtain calibration sources in real systems. Alternatively, another kind of array calibration methods, called auto-calibration, is more preferable, since it does not require calibration sources while the DOAs and other parameters such as mutual coupling coefficients can be estimated simultaneously. The classical mutual coupling autocalibration method proposed by Friedlander et al [4] and the recent one by Sellone et al [5] are able to estimate the DOAs and mutual coupling coefficients using an iterative procedure. However, since a large number of unknown parameters are involved in these two methods, their high computational complexities may be undesired for real-time applications and the convergence may not be guaranteed [6-7].

In order to overcome the drawbacks of mutual coupling auto-calibration methods described above, recent attention has been focused on simplified methods with lower complexities. In [8], mutual coupling calibration method for uniform linear array (ULA) is presented. This method is based on the fact that the mutual coupling coefficient between two sensor elements is inversely related to their separation and can be approximated as zero when they are separated by a few wavelengths. Hence, the number of unknown parameters is reduced significantly. To adopt the MUSIC algorithm directly for DOA estimation, only the middle subarray is utilized.

In this paper, we present a new method for DOA estimation for ULAs in the presence of mutual coupling. The symmetric Toeplitz mutual coupling matrix (MCM) of an ULA as in [8] is employed. Unlike the method in [8], we propose to use the whole array, instead of the middle subarray, to improve the accuracy of DOA estimation. The proposed method is based on the subspace principle, where the DOAs of incoming signals and the mutual coupling coefficients can be estimated jointly. Simulation results show that the proposed algorithm has a better performance than the method in [8], especially for weak signals.

\section{PROBLEM FORMULATION}

Consider a ULA with $M$ sensors impinged by $N$ narrowband signals $s_{n}(t)$ from unknown directions $\theta_{n}$, where $n=1,2, \cdots, N$. Assuming all sensors are identical and no other errors such as location uncertainties are present, the ideal steering vector at an angle $\theta$ can be obtained from the array geometry as

$$
\boldsymbol{a}(\theta)=\left[1, \beta(\theta), \cdots, \beta(\theta)^{M-1}\right]^{\mathrm{T}},
$$

where $\beta(\theta)=\exp \left(-j 2 \pi \lambda^{-1} d \sin \theta\right), d$ is the inter-sensor spacing, $\lambda$ is the signal carrier wavelength and superscript $\mathrm{T}$ denotes matrix transposition. The vector of observed array 
output can be written as

$$
\boldsymbol{x}(t)=\boldsymbol{A s}(t)+\boldsymbol{n}(t),
$$

where $\boldsymbol{A}=\left[\boldsymbol{a}\left(\theta_{1}\right), \boldsymbol{a}\left(\theta_{2}\right), \cdots, \boldsymbol{a}\left(\theta_{N}\right)\right]$ is the ideal steering matrix, $\boldsymbol{s}(t)=\left[s_{1}(t), s_{2}(t), \cdots, s_{N}(t)\right]^{\mathrm{T}}$ is the vector of signal waveforms, respectively, and $\boldsymbol{n}(t)$ is an independent and identically distributed (i.i.d.) additive white Gaussian noise (AWGN) vector with zero mean and covariance matrix $\sigma^{2} \boldsymbol{I}$.

In practice, the interaction between the sensors will result in mutual coupling, which distorts the ideal steering vector significantly. In these situations, the true steering vector should be rewritten as

$$
a_{m}(\theta)=\boldsymbol{C a}(\theta),
$$

where $\boldsymbol{C}$ is the MCM. It is known that, the mutual coupling coefficient between two sensors is inversely related to their distance, and thus the mutual coupling coefficient can be approximated to zero for two sensors which are separated by several sensors far away. More precisely, when the distance between two sensors is more than $P$ inter-sensor spacings, the mutual coupling coefficients can be approximated to be zero. As a result, the resultant MCM can be sufficiently modeled as a banded symmetric Toeplitz matrix as follows [8]

$$
\boldsymbol{C}=\left[\begin{array}{ccccccccc}
1 & c_{1} & \cdots & c_{P-1} & & & & & \\
c_{1} & 1 & c_{1} & \cdots & c_{P-1} & & & \mathbf{0} & \\
\vdots & \ddots & \ddots & \ddots & \vdots & \ddots & & & \\
c_{P-1} & \cdots & c_{1} & 1 & c_{1} & \cdots & c_{P-1} & & \\
& \ddots & \vdots & \ddots & \ddots & \ddots & \vdots & \ddots & \\
& & c_{P-1} & \cdots & c_{1} & 1 & c_{1} & \cdots & c_{P-1} \\
& & & \ddots & \vdots & \ddots & \ddots & \ddots & \vdots \\
& 0 & & & c_{P-1} & \cdots & c_{1} & 1 & c_{1} \\
& & & & & c_{P-1} & \cdots & c_{1} & 1
\end{array}\right]_{M \times M}
$$

Replacing $\boldsymbol{a}(\theta)$ into (2) with $\boldsymbol{a}_{\boldsymbol{m}}(\theta)$, the array output can then be represented by the following model

$$
\boldsymbol{x}(t)=\boldsymbol{C A s}(t)+\boldsymbol{n}(t) .
$$

The array covariance matrix is given by

$$
\boldsymbol{R}_{X}=E\left[\boldsymbol{x}(t) \boldsymbol{x}^{\mathrm{H}}(t)\right]=\boldsymbol{C} \boldsymbol{A} \boldsymbol{R}_{S} \boldsymbol{A}^{\mathrm{H}} \boldsymbol{C}^{\mathrm{H}}+\boldsymbol{\sigma}^{2} \boldsymbol{I},
$$

where the superscript $\mathrm{H}$ denotes the Hermitian transpose operation, $\boldsymbol{R}_{S}=E\left[\boldsymbol{s}(t) \boldsymbol{s}^{\mathrm{H}}(t)\right]$ is the signal covariance matrix. Assuming the $N$ signals are uncorrelated, the rank of $\boldsymbol{R}_{S}$ is $N$. Therefore, the eigenvalue decomposition (EVD) of $\boldsymbol{R}_{X}$ is

$$
\boldsymbol{R}_{X}=\boldsymbol{U}_{S} \boldsymbol{\Sigma}_{S} \boldsymbol{U}_{S}^{\mathrm{H}}+\boldsymbol{U}_{N} \boldsymbol{\Sigma}_{N} \boldsymbol{U}_{N}^{\mathrm{H}} .
$$

where $\boldsymbol{U}_{S} \in \boldsymbol{C}^{M \times N}$ and $\boldsymbol{U}_{N} \in \boldsymbol{C}^{M \times(M-N)}$ are the signal subspace and noise subspace, respectively, $\boldsymbol{\Sigma}_{S} \in \boldsymbol{R}^{N}$ and $\boldsymbol{\Sigma}_{N} \in \boldsymbol{R}^{M-N}$ are diagonal matrices related to the signal and noise power, respectively.

\section{DOA ESTIMATION AND MUTUAL COUPLING COMPENSATION}

In order to calculate the MCM and estimate the DOAs of incoming signals, the authors in [8] used the middle subarray to estimate the DOAs and then estimated the MCM for the whole array. In this section, we will show that, by taking advantage of the symmetric Toeplitz structure of MCM, the whole array can be used to estimate the DOAs and compensate for the mutual coupling effect. This gives a better performance than using the middle subarray, especially for signals with low signal to noise ratio (SNR). According to the MCM and signal models above, the steering vector of the array can be re-written as

$$
\boldsymbol{a}_{\boldsymbol{m}}(\theta)=\left[1+\sum_{i=1}^{P-1} c_{i}\left(\beta(\theta)^{i}+\beta(\theta)^{-i}\right)\right] \beta(\theta)^{P-1} \Gamma(\theta) \boldsymbol{a}(\theta)
$$

where

$$
\Gamma(\theta)=\operatorname{diag}\left[\begin{array}{lllllllll}
\mu_{1} & \cdots & \mu_{P-1} & 1 & \cdots & 1 & \alpha_{1} & \cdots & \alpha_{P-1}
\end{array}\right]
$$

is a diagonal matrix containing $M-2 P+2$ ones between the entry $\mu_{P-1}$ and $\alpha_{1}$, and

$$
\mu_{k}=\frac{\beta(\theta)^{P-1}+\sum_{i=1}^{k-1} c_{i} \beta(\theta)^{P-1-i}+\sum_{i=1}^{P-1} c_{i} \beta(\theta)^{P-1+i}}{\beta(\theta)^{P-1}+\sum_{i=1}^{P-1} c_{i} \beta(\theta)^{P-1-i}+\sum_{i=1}^{P-1} c_{i} \beta(\theta)^{P-1+i}},
$$

and

$$
\alpha_{k}=\frac{\beta(\theta)^{P-1}+\sum_{i=1}^{P-1} c_{i} \beta(\theta)^{P-1-i}+\sum_{i=1}^{P-1-k} c_{i} \beta(\theta)^{P-1+i}}{\beta(\theta)^{P-1}+\sum_{i=1}^{P-1} c_{i} \beta(\theta)^{P-1-i}+\sum_{i=1}^{P-1} c_{i} \beta(\theta)^{P-1+i}},
$$

where, $1 \leq k \leq P-1$. For notational simplification, we define $\sum_{i=1}^{0} f(i)=0$, where $f(i)$ is a function of $i$. From (9), it can be noted that mutual coupling can be viewed as angularly dependent array gain and phase uncertainties. However, it is in general difficult to calibrate the angular dependent array gain and phase uncertainties to account for the mutual coupling. Interestingly, there is a string of ones in the diagonal matrix $\Gamma(\theta)$, indicating that the middle subarray can be considered as an array without gain and phase uncertainties. We now show that it is possible to jointly estimate the DOAs and mutual coupling coefficients based on this property.

Since $\Gamma(\theta)$ is a diagonal matrix with $M-2 P+2$ ones and $\boldsymbol{a}(\theta)$ is a column vector, (8) can be re-written as

$$
\boldsymbol{a}_{\boldsymbol{m}}(\theta)=\left[1+\sum_{i=1}^{P-1} c_{i}\left(\beta(\theta)^{i}+\beta(\theta)^{-i}\right)\right] \beta(\theta)^{P-1} \boldsymbol{T}(\theta) \alpha,
$$

where

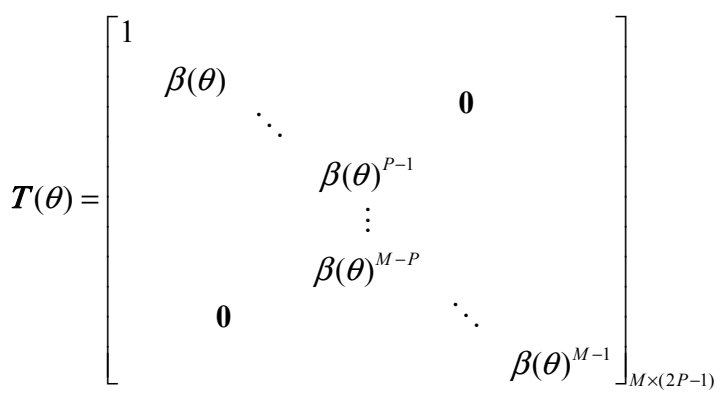

is an $M \times(2 P-1)$ matrix and

$$
\alpha=\left[\begin{array}{lllllll}
\mu_{1} & \cdots & \mu_{P-1} & 1 & \alpha_{1} & \cdots & \alpha_{P-1}
\end{array}\right]^{\mathrm{T}}
$$

is a $(2 P-1) \times 1$ vector with the $P$-th entry equal to 1 .

As mentioned in section II, the signal subspace $\boldsymbol{U}_{S}$ and the noise subspace $\boldsymbol{U}_{N}$ can be obtained from the EVD of covariance matrix $\boldsymbol{R}_{X}$ in (7). From the principle of subspace, 
the steering vector of the incoming signal is orthogonal to the noise subspace. Hence, we have

$$
\boldsymbol{a}_{\boldsymbol{m}}^{\mathrm{H}}(\theta) \boldsymbol{U}_{N} \boldsymbol{U}_{N}^{\mathrm{H}} \boldsymbol{a}_{\boldsymbol{m}}(\theta)=0 .
$$

Generally, $\quad 1+\sum_{i=1}^{P-1} c_{i}\left(\beta(\theta)^{i}+\beta(\theta)^{-i}\right)$ is assumed to be nonzero. Then, substituting (12) into (15), one gets

$$
\begin{gathered}
\boldsymbol{\alpha}^{\mathrm{H}} \boldsymbol{Q}(\theta) \boldsymbol{\alpha}=0, \\
\boldsymbol{Q}(\theta)=\boldsymbol{T}(\theta){ }^{\mathrm{H}} \boldsymbol{U}_{N} \boldsymbol{U}_{N}^{\mathrm{H}} \boldsymbol{T}(\theta)
\end{gathered}
$$

is a $(2 P-1) \times(2 P-1)$ matrix. As the number of signals is $N$ and the number of sensors is $M$, the rank of $\boldsymbol{U}_{n} \boldsymbol{U}_{n}^{\mathrm{H}}$ is $(M-N)$, i.e., $\operatorname{rank}\left[\boldsymbol{U}_{N} \boldsymbol{U}_{N}^{\mathrm{H}}\right]=M-N$. Under the assumption that $2 P-1 \leq M-N$, i.e. $N \leq M-2 P+1, \boldsymbol{Q}(\theta)$ is of full rank for a general $\theta$. However, $\boldsymbol{Q}(\theta)$ is rank deficiency if and only if $\theta$ is equal to one of the $N$ desired DOAs, i.e. $\theta=\theta_{n}(n=1,2, \cdots, N)$ and its determinant is equal to zero. Then the DOAs of signals can be estimated as

$$
\hat{\theta}=\arg \max _{\theta}\{\operatorname{det}[\boldsymbol{Q}(\theta)]\}^{-1},
$$

where $\operatorname{det}[\cdot]$ denotes determinant of a matrix. On the other hand, the smallest eigenvalue of $\boldsymbol{Q}(\theta)$ is also equal to zero when $\theta=\theta_{n}(n=1,2, \cdots, N)$. Then the DOAs can also be estimated based on the smallest eigenvalue of $\boldsymbol{Q}(\theta)$ as

$$
\hat{\theta}=\arg \max _{\theta} \lambda_{\min }^{-1}[\boldsymbol{Q}(\theta)],
$$

where $\lambda_{\min }[\cdot]$ denotes the smallest eigenvalue of a matrix. Since only a determinant operation (or EVD) and onedimensional search are required for the proposed DOA estimation, the computational load is comparable to that in [8] and lower than those involved convergence burden in [4-5].

It can be seen that (16) is satisfied when $\alpha$ is the eigenvector corresponding to the smallest eigenvalue of $\boldsymbol{Q}(\hat{\theta})$, which is denoted $\boldsymbol{r}_{\min }$ here. Considering the condition that the $P$-th entry of $\alpha$ equal to 1 , then $\alpha$ can be estimated as

$$
\hat{\boldsymbol{\alpha}}=\boldsymbol{r}_{\min } \text { with }\left[\boldsymbol{r}_{\min }\right]_{P}=1,
$$

where $\left[\boldsymbol{r}_{\min }\right]_{P}$ denotes the $P$-th entry of vector $\boldsymbol{r}_{\min }$. From (11), (14) and (20), it is known that the mutual coupling coefficients are embedded in $\alpha$. We now proceed to $c$. First of all, define

$$
\begin{gathered}
\boldsymbol{c}=\left[\begin{array}{llll}
c_{1} & c_{2} & \cdots & c_{P-1}
\end{array}\right]^{\mathrm{T}}, \\
\boldsymbol{v}=\left[\begin{array}{lllll}
v_{1} & \cdots & v_{k} & \cdots & v_{P-1}
\end{array}\right]^{\mathrm{T}},
\end{gathered}
$$

respectively, where $v_{k}=\left[\boldsymbol{r}_{\text {min }}\right]_{k+P}$ denotes the $k$-th element of $\boldsymbol{v}$. From (11), (20) and (22), we know that, for any $k \in[1, P-1]$, we have $\hat{\alpha}_{k}=v_{k}$, then

$$
\begin{aligned}
\left(1-v_{k}\right) \sum_{i=1}^{P-1} & c_{i} \beta(\hat{\theta})^{P-1-i}+\sum_{i=1}^{P-1-k} c_{i} \beta(\hat{\theta})^{P-1+i} \\
& \quad-v_{k} \sum_{i=1}^{P-1} c_{i} \beta(\hat{\theta})^{P-1+i}=\left(v_{k}-1\right) \beta(\hat{\theta})^{P-1} .
\end{aligned}
$$

The equation above can be also written in vector form as

$$
\left[\left(1-v_{k}\right) \beta_{1}(\hat{\theta})+\beta_{2 k}(\hat{\theta})-v_{k} \beta_{3}(\hat{\theta})\right] c=\left(v_{k}-1\right) \beta(\hat{\theta})^{P-1},
$$

where

$$
\begin{gathered}
\beta_{1}(\hat{\theta})=\left[\begin{array}{llll}
\beta(\hat{\theta})^{P-2} & \beta(\hat{\theta})^{P-3} & \cdots & 1
\end{array}\right], \\
\beta_{2 k}(\hat{\theta})=\left[\begin{array}{llll}
\beta(\hat{\theta})^{P} & \cdots & \beta(\hat{\theta})^{2(P-1)-k} & \mathbf{0}_{k}
\end{array}\right], \\
\beta_{3}(\hat{\theta})=\left[\begin{array}{llll}
\beta(\hat{\theta})^{P} & \beta(\hat{\theta})^{P+1} & \cdots & \beta(\hat{\theta})^{2(P-1)}
\end{array}\right]
\end{gathered}
$$

are $1 \times(P-1)$ vectors, and $\mathbf{0}_{k}$ is $1 \times k$ zero vector. Denote

$$
\begin{gathered}
\boldsymbol{f}_{k}=\left[\left(1-v_{k}\right) \boldsymbol{\beta}_{1}(\hat{\theta})+\boldsymbol{\beta}_{2 k}(\hat{\theta})-v_{k} \boldsymbol{\beta}_{3}(\hat{\theta})\right], \\
g_{k}=\left(v_{k}-1\right) \beta(\hat{\theta})^{P-1},
\end{gathered}
$$

since $1 \leq k \leq P-1$, (24) can be extended to form

$$
\left[\begin{array}{lll}
\boldsymbol{f}_{1}^{\mathrm{T}} & \cdots & \boldsymbol{f}_{P-1}^{\mathrm{T}}
\end{array}\right]^{\mathrm{T}} \boldsymbol{c}=\left[\begin{array}{lll}
g_{1} & \cdots & g_{P-1}
\end{array}\right]^{\mathrm{T}} .
$$

Therefore, the mutual coupling vector $c$ can be estimated by solving (30) with a general estimated DOA $\theta$ as

$$
\boldsymbol{c}=\boldsymbol{F}^{-1} \boldsymbol{G},
$$

where $\boldsymbol{F}=\left[\begin{array}{lll}\boldsymbol{f}_{1}^{\mathrm{T}} & \cdots & \boldsymbol{f}_{P-1}^{\mathrm{T}}\end{array}\right]^{\mathrm{T}}$ is a $(P-1) \times(P-1)$ matrix, and $\boldsymbol{G}=\left[\begin{array}{lll}g_{1} & \cdots & g_{P-1}\end{array}\right]^{\mathrm{T}}$ is a $(P-1) \times 1$ vector.

In order to get a better performance, all of the estimated DOAs will be applied to calculate the mutual coupling coefficients. We extend (30) with $N$ estimated DOAs as

$$
\overline{\boldsymbol{F}} \boldsymbol{c}=\overline{\boldsymbol{G}},
$$

where $\overline{\boldsymbol{F}}=\left[\begin{array}{lll}\boldsymbol{F}_{1}^{\mathrm{T}} & \cdots & \boldsymbol{F}_{N}^{\mathrm{T}}\end{array}\right]^{\mathrm{T}}, \overline{\boldsymbol{G}}=\left[\begin{array}{lll}\boldsymbol{G}_{1}^{\mathrm{T}} & \cdots & \boldsymbol{G}_{N}^{\mathrm{T}}\end{array}\right]^{\mathrm{T}}, \boldsymbol{F}_{n}$ and $\boldsymbol{G}_{n}$ represent $\boldsymbol{F}$ and $\boldsymbol{G}$ corresponding to the $n$-th estimated DOA $\hat{\theta}_{n}$, respectively. Solving (32), one gets

$$
\boldsymbol{c}=\left(\overline{\boldsymbol{F}}^{\mathrm{H}} \overline{\boldsymbol{F}}\right)^{-1} \overline{\boldsymbol{F}}^{\mathrm{H}} \overline{\boldsymbol{G}} .
$$

Moreover, the performance can be further improved with iterations and an increasing computational load. More precisely, once the estimate of $c$ is obtained, the MUSIC algorithm with the estimated mutual coupling can be implemented, and a more precise estimate of DOAs can be obtained from the MUSIC spatial spectrum as

$$
P_{\text {MUSIC }}=\left\|\boldsymbol{U}_{N}^{\mathrm{H}} \hat{\boldsymbol{C}} \boldsymbol{a}(\theta)\right\|^{-2} .
$$

Then, the mutual coupling coefficients $c$ can be re-computed using the new estimates of DOA from (34). Such procedure can be iteratively repeated to enhance the performance. The simulation results in the next section shows that a satisfactory performance can be obtained with only one iteration.

\section{Simulation ReSUltS}

Consider a ULA with 10 sensors separated by half wavelength, i.e. $d=0.5 \lambda$. The mutual coupling is assumed to be negligible at a distance large than $1.5 \lambda$. Hence, $P=3$ and the coefficients are assumed to be $c_{1}=0.7 e^{j \pi / 6}$, $c_{2}=0.3 e^{-j \pi / 10}$. Two uncorrelated narrow-band signals with equal power impinge on the array from the far-field with directions $10^{\circ}$ and $30^{\circ}$ and 500 snapshots are obtained. Fig. 1 


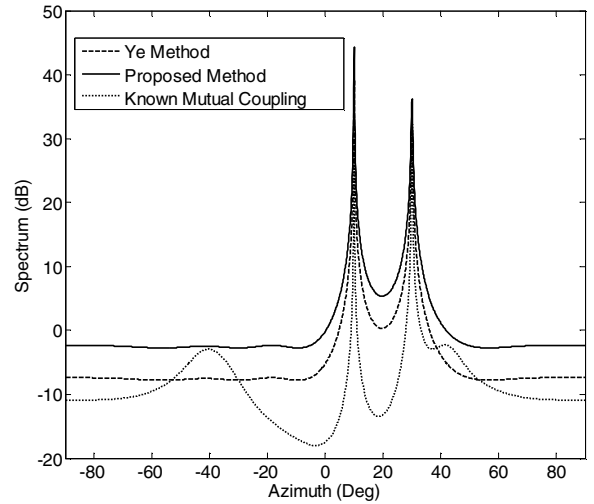

Fig.1 Spectrums with 500 snapshots, SNR=10dB.

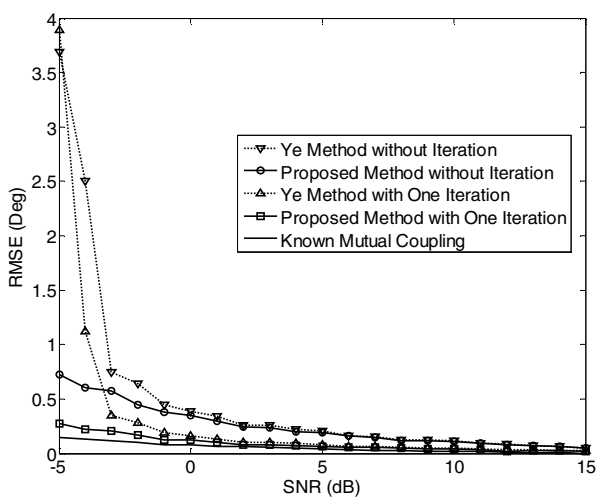

Fig. 2 The RMSE of DOA versus SNR.

shows the spatial spectrums obtained using the proposed method and the method in [8] with a SNR of $10 \mathrm{~dB}$. As a comparison, the MUSIC algorithm with known mutual coupling is also included in Fig. 1. It can be seen that both methods work well at the SNR of $10 \mathrm{~dB}$.

Next, 100 Monte-Carlo experiments are carried out using a set of different SNR levels from $-5 \mathrm{~dB}$ to $15 \mathrm{~dB}$. The root mean squared error (RMSE) criterion is employed to assess and compare the DOA estimation results of different algorithms in a quantitative manner, and it is calculated as $\mathrm{RMSE}=\sqrt{\sum_{k=1}^{K} \sum_{n=1}^{N}\left(\theta_{n}-\hat{\theta}_{k, n}\right)^{2} /(K N)}$, where $K$ is the number of Monte Carlo experiments, $N$ is the number of signals, $\theta_{n}$ is the $n$-th DOA and $\hat{\theta}_{k, n}$ denotes the $n$-th estimated DOA in the $k$-th Monte Carlo experiment. Fig. 2 shows the RMSE versus the SNR curves obtained using the two methods with and without iteration. It can be seen that without iteration the proposed method, which uses the whole array to estimate the DOA, outperforms the method in [8] at low SNR levels. Similar results can be observed for the accuracies of the estimated mutual coupling coefficients. As shown in Tables 1 and 2, where the values are obtained from the averaged estimations of the 100 experiments, the proposed method achieves a more accurate estimate of $c_{1}$ for small SNR. The superiority of the proposed method diminishes with the increase of SNR, and when the SNR is larger than $5 \mathrm{~dB}$, both methods have comparable performance. On the other hand, it can also be seen in Fig. 2 that the performances of both
Table 1. Estimated amplitude of $c_{1}$ against SNR (True value $\rho_{1}=0.7$ ).

\begin{tabular}{|c|c|c|c|c|}
\hline & \multicolumn{2}{|c|}{ Ye Method [8] } & \multicolumn{2}{c|}{ Proposed Method } \\
\hline SNR & $\hat{\rho}_{1}$ & $\left|\rho_{1}-\hat{\rho}_{1}\right|$ & $\hat{\rho}_{1}$ & $\left|\rho_{1}-\hat{\rho}_{1}\right|$ \\
\hline$-5 \mathrm{~dB}$ & 0.4416 & 0.2584 & 0.6249 & 0.0751 \\
\hline$-3 \mathrm{~dB}$ & 0.6276 & 0.0728 & 0.6663 & 0.0337 \\
\hline$-1 \mathrm{~dB}$ & 0.6741 & 0.0259 & 0.6780 & 0.0220 \\
\hline $1 \mathrm{~dB}$ & 0.6781 & 0.0219 & 0.6904 & 0.0096 \\
\hline $3 \mathrm{~dB}$ & 0.6924 & 0.0076 & 0.6915 & 0.0085 \\
\hline $5 \mathrm{~dB}$ & 0.6841 & 0.0159 & 0.6906 & 0.0094 \\
\hline
\end{tabular}

Table 2. Estimated phase of $c_{1}$ against SNR (True value $\phi_{1}=0.5236$ ).

\begin{tabular}{|c|c|c|c|c|}
\hline & \multicolumn{2}{|c|}{ Ye Method [8] } & \multicolumn{2}{c|}{ Proposed Method } \\
\hline \multirow{2}{*}{ SNR } & $\hat{\phi}_{1}$ & $\left|\phi_{1}-\hat{\phi}_{1}\right|$ & $\hat{\phi}_{1}$ & $\left|\phi_{1}-\hat{\phi}_{1}\right|$ \\
\hline$-5 \mathrm{~dB}$ & 0.6897 & 0.1661 & 0.5411 & 0.0175 \\
\hline$-3 \mathrm{~dB}$ & 0.5458 & 0.0222 & 0.5336 & 0.0100 \\
\hline$-1 \mathrm{~dB}$ & 0.5190 & 0.0046 & 0.5162 & 0.0074 \\
\hline $1 \mathrm{~dB}$ & 0.5286 & 0.0050 & 0.5178 & 0.0058 \\
\hline $3 \mathrm{~dB}$ & 0.5191 & 0.0045 & 0.5236 & 0.0000 \\
\hline $5 \mathrm{~dB}$ & 0.5301 & 0.0065 & 0.5270 & 0.0034 \\
\hline
\end{tabular}

methods can be improved after one iteration, yet the proposed method still offers a better performance than the method in [8] at low SNR.

\section{CONCLUTION}

In this paper, a method for DOA estimation in the presence of mutual coupling was developed. The MCM is modeled as a banded symmetric Toeplitz matrix due to the fact that the mutual coupling between two sensors is negligible when they are separated by several wavelengths. Then the mutual coupling is converted to angularly dependent array gain and phase uncertainties. Based on such a property, the DOAs and mutual coupling coefficients can be estimated simultaneously by reconstructing the steering vector to a matrix as (13). The simulation results demonstrate the effectiveness and advantage of the proposed method in DOA estimation in the presence of mutual coupling.

\section{REFERENCES}

[1] R. O. Schmidt, "Multiple emitter location and signal parameter estimation," IEEE Trans. Antennas Propag., vol. 34, no. 3, pp. 276280, 1986.

[2] R. Roy and T. Kailath, "ESPRIT-estimation of signal parameters via rotational invariance techniques," IEEE Trans. Acoust. Speech Signal Process., vol. 37, no. 7, pp. 984-995, 1989.

[3] B. C. Ng and C. M. S. See, "Sensor-array calibration using a maximum-likelihood approach," IEEE Trans. Antenna Propag., vol. 44, no. 6, pp. 827-835, 1996.

[4] B. Friedlander and A. J. Weiss, "Direction finding in the presence of mutual coupling," IEEE Trans. Antennas Propag., vol. 39, no. 3, pp. 273-284, 1991.

[5] F. Sellone and A. Serra, "A novel online mutual coupling compensation algorithm for uniform and linear arrays," IEEE Trans. Signal Process., vol. 55, no. 2, pp. 560-573, 2007.

[6] Z. Ye, J. Dai, X. Xu and X. Wu, "DOA Estimation for Uniform Linear Array with Mutual Coupling," IEEE Trans. Aerosp. Electron. Syst., vol. 45, no. 1, pp. 280-288, 2009.

[7] E. K. L. Hung, "A critical study of a self-calibration direction-finding method for arrays," IEEE Trans. Signal Process., vol. 42, no. 2, pp. 471-474, 1994.

[8] Z. Ye and C. Liu, "On the resiliency of MUSIC direction finding against antenna sensor coupling," IEEE Trans. Antennas Propag., vol. 56, no. 2, pp. 371-380, 2008. 